\title{
Subject Received Unplanned Treatment
}

National Cancer Institute

\section{Source}

National Cancer Institute. Subject Received Unplanned Treatment. NCI Thesaurus. Code C142240.

The subject, who met eligibility criteria, was assigned to an arm but received treatment not associated with any of the planned arms. 